Research report

\title{
Mindreading in individuals with an empathizing versus systemizing cognitive style: An fMRI study
}

\author{
F. Focquaert ${ }^{\mathrm{a}, *}$, M.S. Steven-Wheeler ${ }^{\mathrm{b}, 1}$, S. Vanneste ${ }^{\mathrm{c}}$, K.W. Doron $^{\mathrm{d}}$, S.M. Platek $^{\mathrm{e}}$ \\ a Department of Philosophy and Moral Sciences, Ghent University, Blandijnberg 2, 9000 Gent, Belgium \\ ${ }^{\mathrm{b}}$ Center for Cognitive Neuroscience, Department of Psychological and Brain Sciences, Dartmouth College, Hinman 6045, Hanover, NH 03755, USA \\ ${ }^{c}$ Department of Neurosurgery \& BRAI ${ }^{2}$, University Hospital Antwerp, Wilrijkstraat 10, 2650 Edegem, Belgium \\ ' Sage Center for the Study of Mind, University of California, Santa Barbara, CA 93106-9660, USA \\ e Department of Psychology, Georgia Gwinnett College, Lawrenceville, GA 30043, USA
}

\section{A R T I C L E I N F O}

\section{Article history:}

Received 28 December 2009

Received in revised form 9 August 2010

Accepted 12 August 2010

Available online 20 August 2010

\section{Keywords:}

Mindreading

Empathizing

Systemizing

Simulation

\begin{abstract}
A B S T R A C T
Our fMRI study compares the neural correlates of face-based mindreading in healthy individuals with an empathizing $(n=12)$ versus systemizing cognitive style $(n=12)$. The empathizing group consists of individuals that score high on empathizing and low on systemizing, while the systemizing group consists of individuals with an opposite cognitive pattern. We hypothesize that the empathizing group will show stronger simulation-type neural activity (e.g., in mirror neuron areas, medial prefrontal cortex, anterior cingulate cortex) or simulation-related neural activity (e.g., in areas involved in perspective taking and experiential processing) compared to the systemizing group. As hypothesized, our study reveals that the empathizing group shows significantly stronger activity in mirror neuron areas of the brain, such as the left inferior frontal gyrus and inferior parietal lobe, and in temporal areas involved in perspective taking and autobiographical memory. Moreover, the empathizing group, but not the systemizing group, shows activity in the medial prefrontal cortex and anterior cingulate cortex which have been related to simulation-type neural activity in the brain and are central to mindreading. Also, the systemizing group shows significantly stronger activity in the left parahippocampal gyrus. In conclusion, both the empathizing and systemizing individuals show simulation-type and simulation-related neural activity during face-based mindreading. However, more neural activity indicative of simulation-based processing is seen in the empathizing individuals, while more neural activity indicative of non-simulation-based processing is seen in the systemizing individuals.
\end{abstract}

(c) 2010 Elsevier Inc. All rights reserved.

\section{Introduction}

During the last decade, several cognitive neuroscience studies have provided evidence in favor of simulation theory (ST) accounts of mindreading [22,23,27,48,57]. ST accounts claim that mindreading involves imagining how the world looks from someone else's perspective. The mindreader simulates the mental world of the target (i.e., evokes a similar experiential and/or psychological state) and links this simulated state to a corresponding mental state, which can then be accessed by means of introspection and attributed to the target [27].

\footnotetext{
* Corresponding author. Tel.: +32 4769898 85; fax: +32 92644187. E-mail addresses: farah.focquaert@ugent.be (F. Focquaert), Megan.Steven@Dartmouth.edu (M.S. Steven-Wheeler), sven.vanneste@ua.ac.be (S. Vanneste), K.Doron@psych.ucsb.edu (K.W. Doron), splatek@gmail.com (S.M. Platek).

1 Tel.: +160364698 87
}

Simulation-type neural activity implies common activation in a given brain area (e.g., medial prefrontal cortex for introspection and theory of mind) or in single cells (e.g., mirror neurons in the inferior frontal gyrus) for both self-aspects and other-aspects of certain cognitive tasks. Common activation in single cells is also referred to as mirroring simulation. Common activation in a given brain area that does not involve single cells or mirror neurons (MNs) is referred to as non-mirroring simulation (see [28]). Non-mirroring simulation implies that one subset of neurons in a given brain area codes for the self-aspect of a particular cognitive task, and another subset of neurons for the other-aspect [16]. Neural activity in areas of the brain that contribute to simulation-based mindreading but do not involve common activation (either in a given brain area or single cells), can be described as simulation-related rather than simulation-type (e.g., areas involved in autobiographical memory, and perspective taking).

According to Goldman [27], face-based mindreading relies on simulation-type neural activity. Evidence in favor of such simulation-type neural activity during face-based emotion recognition is provided in a study by Carr et al. [9] on face-based imitation. 
Carr et al.'s [9] results indicate that an action-mirroring mechanism (i.e., the human mirror neuron system (MNS)) is implicated in face-based emotion recognition. The observation and imitation of emotional faces elicited common activation in the premotor face area, the dorsal inferior frontal gyrus (IFG), the superior temporal sulcus (STS), the insula and amygdala. Moreover, several imaging studies on implicit mindreading (i.e., no overt mental state attribution is involved) have shown that self-reported empathy (or an 'empathizing' cognitive style) correlates with simulation-type neural activity (e.g., [35,44,61]). Schulte-Rüther et al. [60] report the same finding for explicit mindreading skills (i.e., involving overt mental state attribution). Schulte-Rüther et al. [60] argue that their findings support the view that MN mechanisms operate during selfand other-related decoding of emotional face expressions. Interestingly, a study by Cheng et al. [10] showed that empathizing skills correlate positively with MN activity, while systemizing skills correlate negatively with MN activity.

Goldman [27] asserts that simulation is the default strategy for face-based mindreading in all normal individuals. Drawing upon the findings from the above studies, we wanted to investigate whether these simulation-based processes differ in empathizing versus systemizing individuals during explicit mindreading. Hence, to further elucidate the neural correlates of mindreading in normal individuals, the current fMRI study compares individuals with an empathizing cognitive style (i.e., high empathizing and low systemizing) to individuals with a systemizing cognitive style (i.e., low empathizing and high systemizing) during face-based mindreading. This is the first fMRI study investigating the difference between empathizing and systemizing cognitive styles during mindreading. We hypothesize that the empathizing group will show more activity indicative of simulation-type and/or simulation-related processing (in line with ST), such as MN activity in the IFG and inferior parietal lobule (IPL), non-mirroring simulation-type activity in the medial prefrontal cortex (MPFC) and anterior cingulate cortex (ACC), and simulation-related activity in the temporal lobes known to be involved in autobiographical memory and perspective taking.

\section{Materials and methods}

\subsection{Questionnaires}

\subsubsection{Empathy questionnaire (EQ)}

The EQ [6] is a validated 60-item (40 assessing empathy and 20 filler control) measure assessing empathy. Responses are given on a 4-point-Likert scale ranging from strongly disagree to strongly agree. Responses were scored in the suggested manner, with participants receiving 0 for a 'non-empathic' response, 1 for a somewhat empathic and 2 for a very empathic response (e.g., for item 25: strongly agree gets 2 points, agree gets 1 point, and disagree and strongly disagree both get 0 points; or for item 46: strongly disagree gets 2 points, disagree gets 1 point, and agree and strongly agree both get 0 points). The total possible score is 80 . Empathizing can be defined as "the drive to identify another's mental states and to respond to these with an appropriate emotion, in order to predict and to respond to the behavior of another person" ([3], p. 820). Unless an individual has a pathologically high level of empathy, individuals with high empathizing skills typically feel comfortable in social settings, can easily relate to others and are able to form close, lasting relationships with others. These individuals are very socially sensitive. In contrast, individuals with low empathizing typically feel uncomfortable during social settings, have difficulty understanding others' thoughts and feelings, and often find it difficult to establish and maintain social relationships. Women typically score higher on the EQ compared to men [1].

\subsubsection{Systemizing questionnaire (SQ)}

The SQ [4] is a validated 60-item (40 assessing systemizing and 20 filler control) measure assessing systemizing. Responses are given on a 4-point-Likert scale ranging from strongly disagree to strongly agree. Responses were scored in the suggested manner, with participants receiving 0 for a 'non-systemizing' response, 1 for a somewhat systemizing response, and 2 for a very systemizing response (e.g., for item 19: strongly agree gets 2 points, agree gets 1 point, and disagree and strongly disagree both get 0 points; or for item 31: strongly disagree gets 2 points, disagree gets 1 point, and agree and strongly agree both get 0 points). The total possible score is 80 . Systemizing can be defined as "the drive to analyze a system in terms of the rules that govern the system, in order to predict the behavior of the system" ([3], p.
Table 1

Descriptive statistics.

\begin{tabular}{cccccr}
\hline & $n$ & Minimum & Maximum & $M$ & \multicolumn{1}{c}{$S D$} \\
\hline \multicolumn{2}{l}{ Systemizing group } & & & & \\
SQ & 12 & 40 & 59 & 47.83 & 6.09 \\
EQ & 12 & 12 & 43 & 30.83 & 8.42 \\
\multicolumn{2}{l}{ Empathizing group } & & & & \\
SQ & 12 & 10 & 38 & 27.92 & 8.91 \\
EQ & 12 & 36 & 70 & 47.00 & 11.86 \\
\hline
\end{tabular}

820). Individuals that score high on systemizing are good at understanding mechanical systems and input-output relations in general. They have the ability to focus on relevant details and ignore irrelevant ones. They are often engineers or scientists [20]. Individuals with low systemizing skills are typically less good at understanding mechanical systems, input-output relations. Men typically score higher on the SQ compared to women [1].

\subsection{3. 'Revised reading the mind in the eyes' test}

This test is a validated advanced theory of mind test involving complex mental states (e.g., social emotions). It is designed to check how well participants can tune in' to others' thoughts and feelings. The participants are presented with a series of 36 photographs of eyes and are asked to choose which word (out of four) best describes what the person in the photograph is thinking or feeling $[1,7]$. In the present fMRI study, participants are asked to choose between two words (see below).

\subsection{Participants}

Twenty-four male volunteers (mean age $=27$ years, range $18-38$ years) were recruited from the Dartmouth student and post-doc pool in mathematics, physics, chemistry, computer science, engineering, environmental science, theatre, etc. $(N=137)$. Participants were screened using the empathy questionnaire (EQ) and systemizing questionnaire (SQ) [1]. Participants were paid $\$ 10$ per hour for their participation in the behavioral part and informed consent was obtained in accordance with the guidelines set forth by the Dartmouth Committee for the Protection of Human Subjects (CHPS protocol \#17772). We selected two groups of 12 participants each that differed in their empathizing-systemizing cognitive style (see Table 1). One group had high systemizing combined with low empathizing (i.e., systemizing group), and the other group displayed an opposite pattern of high or above average empathizing combined with low systemizing (i.e., empathizing group). On the SQ most normal men score about 30 out of 80 , whereas on the EQ most normal men score about 42 out of 80 (for ranges see [1]).

All participants were right-handed, had normal visual acuity, and were screened for history of psychiatric or medical illness. All participants were male to make sure that gender biases in emotional recognition and empathic processing did not confound our findings $[1,42]$. Participants were paid $\$ 10$ per hour or given T-points for their participation in the imaging study and gave informed consent in accordance with the guidelines set forth by the Dartmouth Committee for the Protection of Human Subjects.

\subsection{Stimuli and experimental design}

All stimuli were shown using NeuroBehavioral Systems Presentation 9.9 (http://www.neurobs.com/) software and consisted of 36 photographs that were part of the 'Revised Reading the Mind in the Eyes' test [1,7]. The 'Reading the Mind in the Eyes' test [2] was previously used in an fMRI study to compare normal individuals to individuals with Asperger syndrome [5]. Baron-Cohen et al. [5] used a force choice task between two words that either indicated an emotional content or the exact opposite of this emotional content. For example, their test used "concerned" versus "unconcerned" ([5], p. 1893) as a forced choice option. Since our participants were all graduate students and might not have been motivated by the relatively easy Baron-Cohen et al. [5] version, we opted for a slightly altered version of the 'Revised Mind in the Eyes' test. Participants could choose between two options with varying emotional content. For example, our test used "hostile" versus "preoccupied." We decided not to include four options, since this might generate too much eye movement on behalf of the participants and result in overlapping frontal activations that might compromise our results. The false options were randomly chosen from the behavioral 'Revised Mind in the Eyes' test consisting of four options each [1].

A blocked CACBCA... design was used. Both task A (control) and task B (face-based mindreading) consisted of a forced choice between two words (either male/female or a choice between two words depicting various mental states). Participants were asked to indicate their response by means of button presses (pressing one of two buttons with the right hand and choosing between the right word and the left word). Correct words were counterbalanced across the right and the left side. During task A, participants were shown 36 photographs of eyes and asked to indicate the gender of the individuals in the photograph. Stimuli were shown for $5.25 \mathrm{~s}$ followed by an ISI of $.75 \mathrm{~s}$. During task B, participants saw the same stimuli 


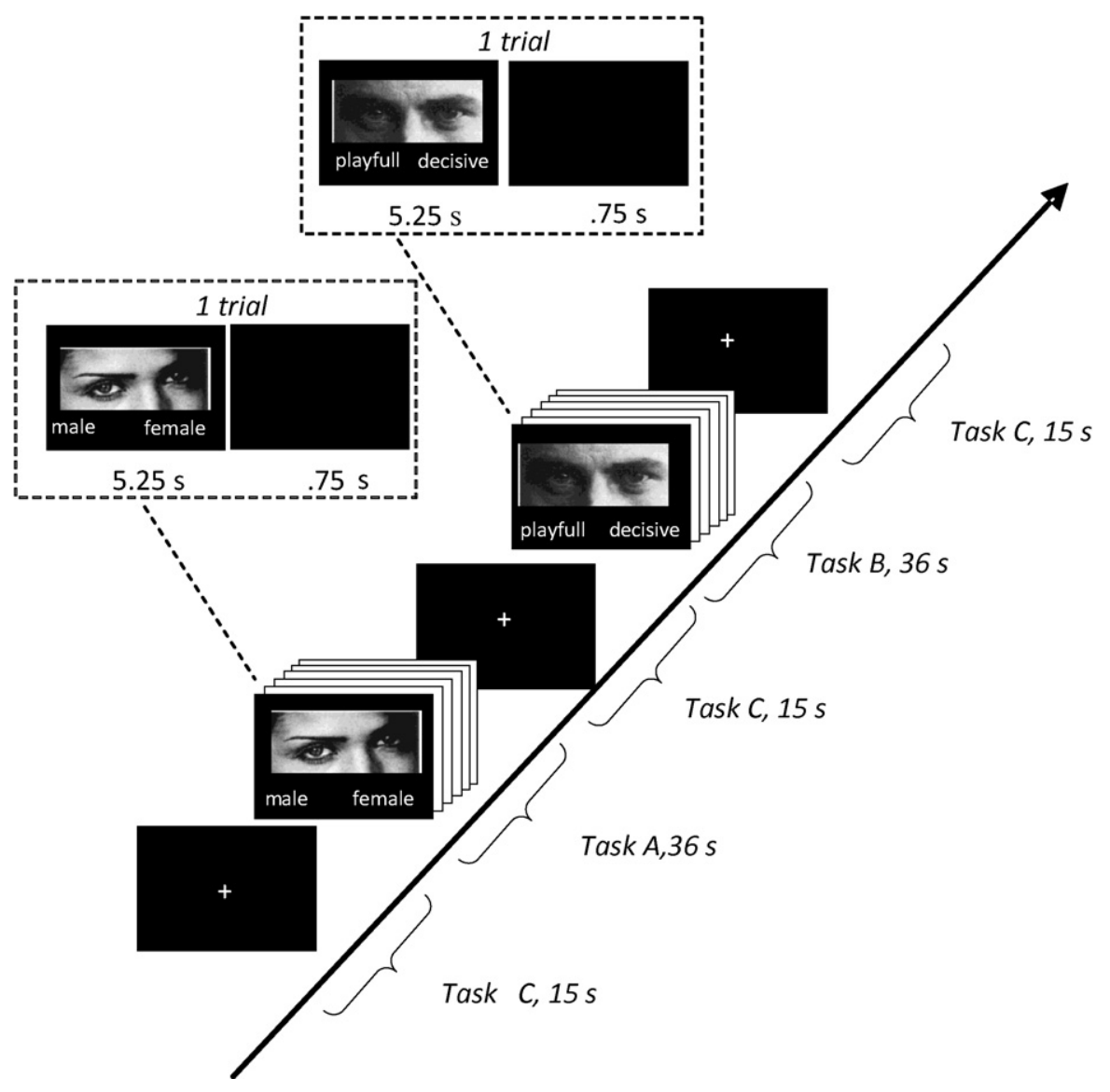

Fig. 1. Overview experimental design.

and were asked to indicate which of two words best described the facial expressions displayed in the photographs. Again, stimuli were shown for $5.25 \mathrm{~s}$. followed by an ISI of $.75 \mathrm{~s}$. Task A and B each lasted for $36 \mathrm{~s}$, during which time 6 photographs were projected to the subjects, and were alternated with a baseline condition (task $C$ ) consisting of a crosshair that lasted for $15 \mathrm{~s}$. Participants were asked to fixate on the crosshair whenever it appeared. Each functional run consisted of 7 rest/crosshair conditions of $15 \mathrm{~s}$ each and 6 task conditions of $36 \mathrm{~s}$ each. Each functional run began and ended with a $15 \mathrm{~s}$ rest/crosshair condition and was alternated with a task condition throughout the run, thus the blocks ran as rest, task, rest, task, etc. Previous lab research has shown that subjects start to move, become bored, fall asleep, etc. when individual functional sessions extend beyond go 5-6 min. We divided up functional runs to account for this. There were two functional runs that were alternated across participants (see Fig. 1).

\section{Data collection}

\subsection{Image acquisition}

Imaging was performed on a 3 Tesla Phillips Scanner using a SENSE head coil for signal reception. fMRI was performed using gradient echo, EPI. Two functional runs (each preceded by two leadin dummy scans) were collected consisting of 107 volumes each with the following parameters used: $\mathrm{TR}=3000 \mathrm{~ms}$, $\mathrm{TE}=35 \mathrm{~ms}$, flip angle $=90^{\circ}, \mathrm{FOV}=240 \mathrm{~mm}$, matrix $=80 \times 80 \times 30$; and slice thickness $=4 \mathrm{~mm}$, skip $.5 \mathrm{~mm}$. Functional runs were randomized across subjects. 3D-high resolution sagittal images were collected for each subject using the following parameters: $\mathrm{TR}=9.9 \mathrm{~ms}$, $\mathrm{TE}=4.6 \mathrm{~ms}$, flip angle $=8^{\circ}$, FOV $=240 \mathrm{~mm}$, matrix $=256 \times 256 \times 160$ and slice thickness $=1 \mathrm{~mm}$.

\section{2. fMRI data preprocessing}

Data preprocessing was performed using FMRI Expert Analysis Tool (FEAT) Version 5.63, which is part of FSL (FMRIB's Software
Library, www.fmrib.ox.ac.uk/fsl) [62]. Each subject completed two fMRI runs and the following pre-statistical processing was applied to each run. Motion correction was applied using the middle scan as reference; images were slice-timing corrected and spatial smoothing was applied using a Gaussian kernel of FWHM $6 \mathrm{~mm}$ and mean-based intensity normalization of all volumes performed by the same factor.

Registration to stereotaxic MNI space was carried out using FLIRT $[37,36]$. Time-series statistical analysis was carried out using FILM prewhitening with local autocorrelation correction [66]; highpass temporal filtering (Gaussian-weighted least-squares straight line fitting, with sigma = $102 \mathrm{~s}$ ). The first and second runs for each subject were combined using fixed effects model, by forcing the random effects variance to zero in FMRIB's Local Analysis of Mixed Effects (FLAME). BOLD responses were assumed to be uniform across brain areas and subjects and were modeled by a $36 \mathrm{~s}$ box-car for the gender and mindreading conditions. Parameter estimates from the design matrix were generated for each subject's timeseries data for the task conditions and rest conditions. Six motion parameters were also included in the design matrix. Contrasts of parameter estimates were generated for the following conditions in each subject: gender > mindreading; mindreading > gender. $Z$ statistic images were thresholded using clusters determined by $Z>2.3$ and a corrected cluster significance threshold of $p=.05$ [67]. In order to determine differential activations between the systemizing and empathizing group, within group activation maps for gender $>$ mindreading and gender $<$ mindreading were examined using independent sample $t$-tests with the fixed effects model described above and subjects as a random factor. To ensure that difference maps were not showing statistically significant negative activation differences, all higher-level contrasts of parameter estimates were constrained by masks containing $Z$-scores greater 
than 0. Post hoc region of interest (ROI) analyses were performed by masking between group $Z$-stat differential contrasts (mindreading > gender) with a Brodmann area mask. The ROIs were defined from within the WFU Pickatlas Matlab program, using Brodmann areas from stereotaxic space (see [49]) and then thresholding unpaired $t$-test results at $p=.005$ (uncorrected). The specific regions of interest were chosen based upon recent fMRI studies on implicit face-based mindreading which show that higher empathizing correlates with more activity in MN-areas and MN-related areas (IFG, IPL, STS, anterior insula (AI)) $[9,35,44]$.

\section{Results}

\subsection{Behavioral results}

\subsubsection{Revised Mind in the Eyes and gender recognition}

Both groups scored significantly above chance on both tasks during scanning. The empathizing group (gender: $M=97 \%, S D=2.8$ ) and systemizing group (gender: $M=96 \%, S D=3.2$ ) scored comparable on the gender task. The empathizing group (mindreading: $M=90 \%, S D=5.6$ ) was more accurate than the systemizing group (mindreading: $M=82 \%, S D=7.2$ ) on the mindreading task. A oneway ANOVA was obtained, revealing a significant effect of group (empathizing vs. systemizing) for the mindreading task, with the empathizing group performing better than the systemizing group $(F(1,22)=8.84, p<.01)$, but no significant effect of group for the gender $\operatorname{task}(F(1,22)=.57$, n.s. $)$.

\section{2. fMRI results}

\subsubsection{Differential contrast analysis}

In the remainder of the paper, mindreading > gender refers to the comparison between the mindreading and the gender task in terms of brain activity; empathizing refers to the empathizing group; systemizing refers to the systemizing group; and empathizing > systemizing refers to the comparison between the empathizing and the systemizing group in terms of brain activity, and vice versa for systemizing > empathizing.

4.2.1.1. systemizing: mindreading > gender. The systemizing group shows significant BOLD responses in the left middle/superior temporal gyrus (BA 21, 22) and the left inferior frontal gyrus (BA 45, 47) during the face-based mindreading task (Table 2).

4.2.1.2. empathizing: mindreading > gender. The empathizing group shows significant BOLD responses in the left middle temporal gyrus (BA 21), the left inferior frontal gyrus (BA 44, 45, 47), the left medial frontal gyrus (BA 9), the left superior frontal gyrus (BA 6,10 ) and the left cingulate gyrus (BA 32) during the face-based mindreading task (Table 2).

4.2.1.3. systemizing > empathizing: mindreading > gender. Our analysis reveals significantly stronger BOLD responses in the left middle/superior temporal gyrus (BA 21, 22), the left temporal pole (BA 38), the right superior frontal gyrus (BA 6), the left middle/inferior frontal gyrus (BA 47) and the left parahippocampal gyrus (BA 27) in the systemizing group (Table 2, Fig. 2).

4.2.1.4. empathizing > systemizing: mindreading > gender. Our analysis reveals significantly stronger BOLD responses in the left inferior frontal gyrus (BA 44, 45, 47), the bilateral superior temporal gyri (BA 22), the bilateral middle temporal gyri (BA 21, 22), the bilateral angular gyrus (BA 39), the left inferior parietal lobule/supramarginal gyrus (BA 40), and in the bilateral fusiform gyrus (BA $18,19,37$ ) in the empathizing group (Table 2, Fig. 3 ).
Table 2

Differential contrast analysis.

\begin{tabular}{|c|c|c|c|c|}
\hline Brain regions & BA & $x$ & $y$ & $z^{\mathrm{a}}$ \\
\hline \multicolumn{5}{|l|}{ 1. Mindreading $>$ gender } \\
\hline \multicolumn{5}{|l|}{ Empathizing } \\
\hline \multicolumn{5}{|l|}{ Temporal lobes } \\
\hline L middle temporal gyrus & 21 & -59 & -52 & 6 \\
\hline \multicolumn{5}{|l|}{ Frontal lobes } \\
\hline L inferior frontal gyrus & 44 & -55 & 18 & 16 \\
\hline L inferior frontal gyrus & 45 & -53 & 26 & 6 \\
\hline L inferior frontal gyrus & 47 & -51 & 29 & -3 \\
\hline L medial frontal gyrus & 9 & -4 & 35 & 33 \\
\hline L superior frontal gyrus & 6 & 0 & 17 & 60 \\
\hline L superior frontal gyrus & 10 & -8 & 60 & 25 \\
\hline \multicolumn{5}{|l|}{ Limbic system } \\
\hline L cingulate gyrus & 32 & -8 & 18 & 40 \\
\hline \multicolumn{5}{|l|}{ Systemizing } \\
\hline \multicolumn{5}{|l|}{ Temporal lobes } \\
\hline L middle temporal gyrus & 21 & -61 & -46 & 8 \\
\hline L middle temporal gyrus & 22 & -53 & -39 & -1 \\
\hline L superior temporal gyrus & 22 & -57 & -52 & 10 \\
\hline \multicolumn{5}{|l|}{ Frontal lobes } \\
\hline L inferior frontal gyrus & 45 & -53 & 18 & 19 \\
\hline L inferior frontal gyrus & 47 & -46 & 27 & -10 \\
\hline \multicolumn{5}{|l|}{ 2. Mindreading $>$ gender } \\
\hline \multicolumn{5}{|l|}{ Empathizing $>$ systemizing } \\
\hline \multicolumn{5}{|l|}{ Frontal lobes } \\
\hline L precentral gyrus & 44 & -53 & 10 & 9 \\
\hline L inferior frontal gyrus & 45 & -53 & 35 & 2 \\
\hline L inferior frontal gyrus & 47 & -50 & 25 & -1 \\
\hline \multicolumn{5}{|l|}{ Temporal lobes } \\
\hline $\mathrm{R}$ middle temporal gyrus & 21 & 63 & -50 & 3 \\
\hline $\mathrm{R}$ middle temporal gyrus & 22 & 59 & -47 & 2 \\
\hline $\mathrm{R}$ middle temporal gyrus & 39 & 51 & -66 & 11 \\
\hline L middle temporal gyrus & 21 & -59 & -52 & 6 \\
\hline L middle temporal gyrus & 19 & -55 & -61 & 16 \\
\hline L middle temporal gyrus & 37 & -51 & -60 & 0 \\
\hline L middle temporal gyrus & 39 & -42 & -52 & 14 \\
\hline R superior temporal gyrus & 22 & 50 & -46 & 15 \\
\hline L superior temporal gyrus & 22 & -42 & -52 & 17 \\
\hline L fusiform gyrus & 37 & -40 & -57 & -14 \\
\hline L fusiform gyrus & 37 & -40 & -63 & -12 \\
\hline R fusiform gyrus & 18 & 28 & -85 & -23 \\
\hline R fusiform gyrus & 19 & 26 & -82 & -14 \\
\hline \multicolumn{5}{|l|}{ Parietal lobes } \\
\hline L inferior parietal lobule & 40 & -63 & -40 & 24 \\
\hline L supramarginal gyrus & 40 & -65 & -49 & 23 \\
\hline \multicolumn{5}{|l|}{ Systemizing > empathizing } \\
\hline \multicolumn{5}{|l|}{ Temporal lobes } \\
\hline L middle temporal gyrus & 21 & -59 & -22 & -6 \\
\hline L superior temporal gyrus & 38 & -57 & 11 & -17 \\
\hline \multicolumn{5}{|l|}{ Frontal lobes } \\
\hline R superior frontal gyrus & 6 & 4 & 14 & 56 \\
\hline L superior frontal gyrus & 6 & -2 & 28 & 58 \\
\hline L superior frontal gyrus & 6 & 0 & 7 & 57 \\
\hline L middle frontal gyrus & 47 & -48 & 48 & -9 \\
\hline L inferior frontal gyrus & 47 & -51 & 46 & -14 \\
\hline \multicolumn{5}{|l|}{ Cortical } \\
\hline L parahippocampal gyrus & 27 & -20 & -31 & -7 \\
\hline
\end{tabular}

Note: All results of unpaired $t$-test significant at $p<.05$, cluster-level corrected. BA = Brodmann's area; $\mathrm{L}=$ left, $\mathrm{R}=$ right

a Cluster peak in MNI coordinates.

Local maxima in multiple regions (BA) with the highest $Z$-score within the cluster are reported.

\subsubsection{ROI analysis (mindreading $>$ gender)}

The ROI analysis (see Table 3 , Fig. 4) reveals significantly stronger BOLD responses in the left inferior frontal gyrus (BA 44 ), left superior temporal gyrus (BA 22), bilateral middle temporal gyrus (BA 21, 22) and the left inferior parietal lobule (BA 40 ) in the empathizing group (empathizing > systemizing: min- 


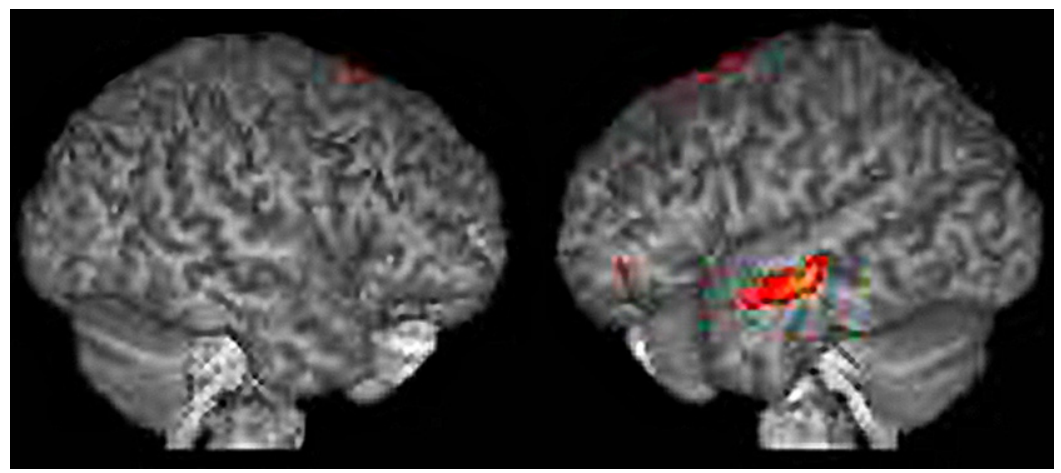

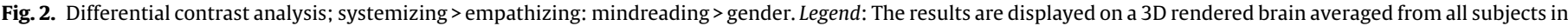

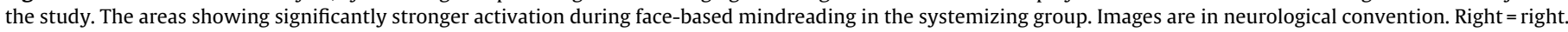
Left = left. RH activation: BA 6. LH activation: BA 21, BA 38, BA 47, BA 27.

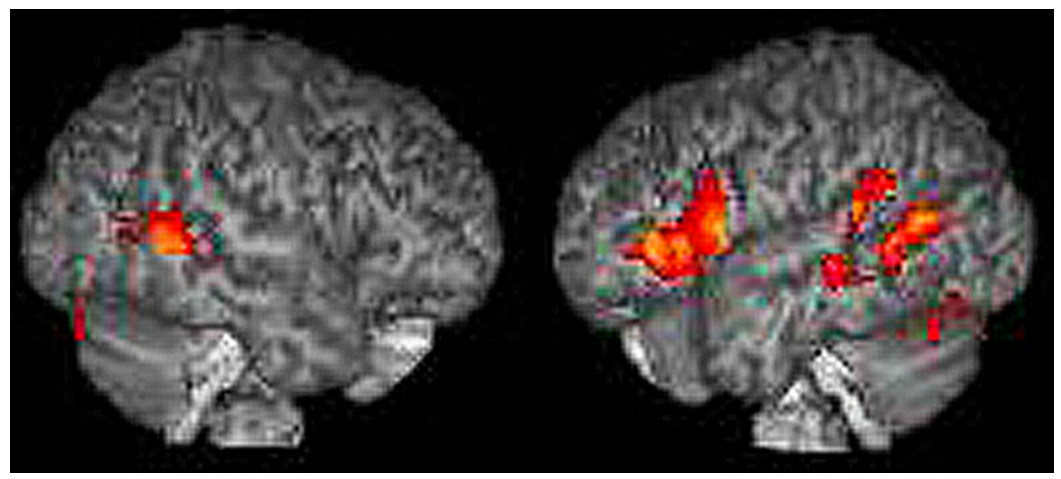

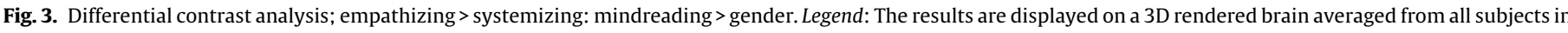

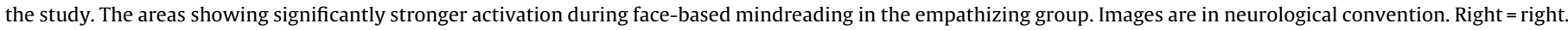
Left = left. LH activation: BA 44, BA 45, BA 47, and BA 40. Bilateral activation: BA 22, BA 21, BA 39, BA 18 , BA 19 and BA 37.

Table 3

ROI analysis.

\begin{tabular}{|c|c|c|c|c|c|c|}
\hline Brain region: mindreading > gender & $\mathrm{BA}$ & $x$ & $y$ & $z^{\mathrm{a}}$ & No. of voxels & Maximum Z-score \\
\hline \multicolumn{7}{|l|}{ Empathizing > systemizing } \\
\hline L inferior frontal gyrus & 44 & -54 & 10 & 10 & 218 & 6.28 \\
\hline L inferior parietal lobule & 40 & -64 & -38 & 34 & 1579 & 5.99 \\
\hline $\mathrm{R}$ middle temporal gyrus & 21 & 64 & -54 & 0 & 1064 & 5.99 \\
\hline $\mathrm{R}$ middle temporal gyrus & 22 & 60 & -48 & 2 & 861 & 5.34 \\
\hline L middle temporal gyrus & 21 & -62 & -54 & -2 & 1032 & 4.79 \\
\hline L superior temporal gyrus & 22 & -60 & -62 & 12 & 842 & 5.45 \\
\hline \multicolumn{7}{|l|}{ Systemizing > empathizing } \\
\hline L inferior frontal gyrus & 44 & & & & & \\
\hline $\mathrm{R}$ inferior parietal lobule & 40 & & & & & \\
\hline R superior temporal gyrus & 21 & 62 & -24 & -4 & 1064 & 5.16 \\
\hline R middle temporal gyrus & 22 & & & & & \\
\hline L middle temporal gyrus & 21 & -62 & -18 & -12 & 1032 & 6.64 \\
\hline L superior temporal gyrus & 22 & -66 & -48 & 10 & 842 & 5.15 \\
\hline
\end{tabular}

Note: All results of unpaired $t$-test significant at $p=.005$, uncorrected. $\mathrm{BA}=$ Brodmann's area; $\mathrm{L}=$ left, $\mathrm{R}=$ right.

a Peak statistical height in MNI coordinates.

dreading > gender). Significantly stronger BOLD responses in the right and left superior temporal (BA 22) and left middle temporal gyrus (BA 21) are found in the systemizing group (systemizing > empathizing: mindreading > gender) (Table 3, Fig. 4). We did not find insula differences.

\section{Discussion}

\subsection{Main findings}

Our study investigates whether normal individuals with an empathizing versus systemizing cognitive style differ in terms of simulation-based neural processes during face-based mindreading. Even though all our participants are normal individuals matched in terms of gender, age, education and socio-economical background, our study reveals significant differences in brain activity between the systemizing group and the empathizing group. Behaviorally, although both groups perform highly above chance, the empathizing group performs better than the systemizing group.

Briefly summarizing our main BOLD results: the general analysis reveals significantly stronger activity in the left IFG (BA 44), bilateral STG (BA 22), the left IPL (BA 40), the bilateral TPJ (BA 39) and the bilateral fusiform gyrus (FG; BA 19,37) in the empathizing 
(A)

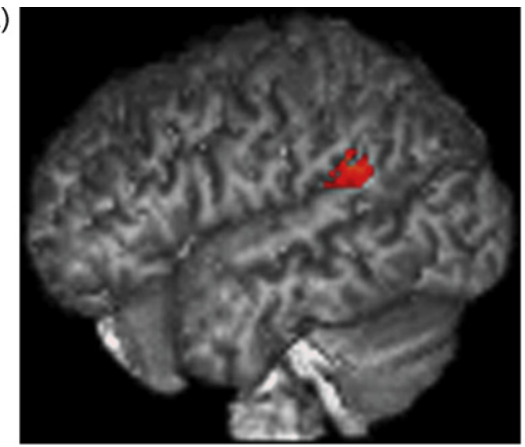

(C)

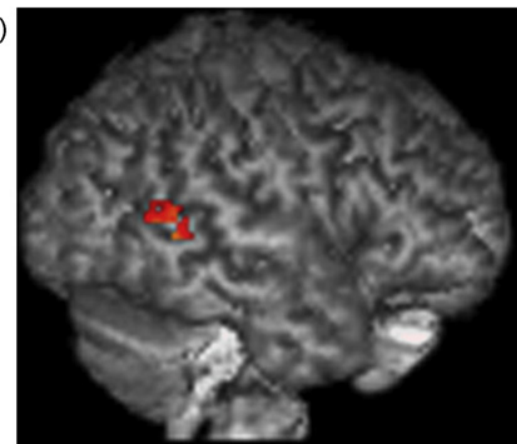

(B)

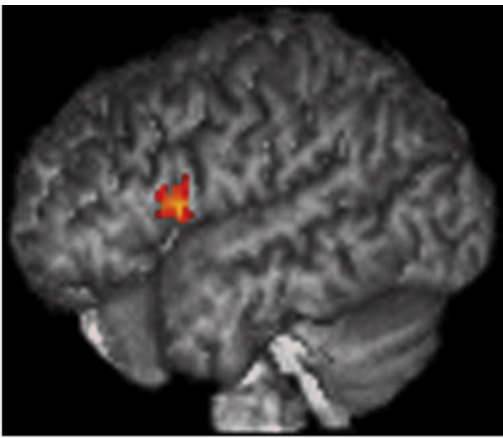

(D)

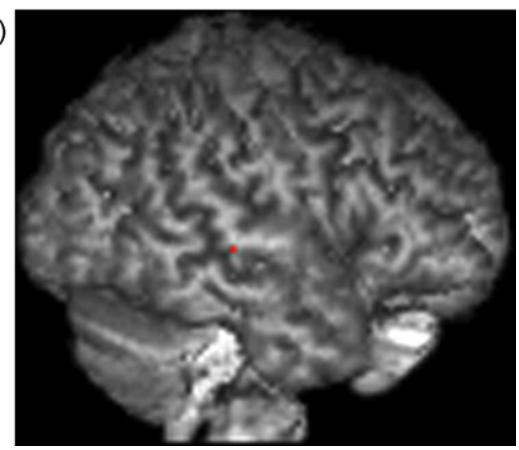

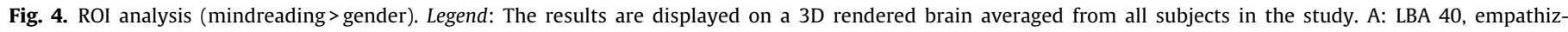

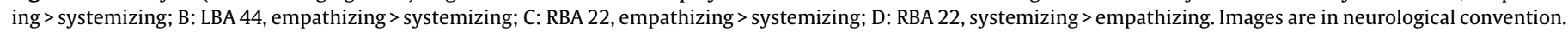
Right $=$ right . Left $=$ left.

group. In the systemizing group, our analysis reveals significantly stronger activity in the left temporal pole (TP; BA 38), the right superior frontal gyrus (SFG; BA 6), and the left parahippocampal gyrus. Moreover, our general analysis shows activity in the MPFC and ACC in the empathizing group but not the systemizing group.

\subsection{The empathizing group}

\subsection{1. $M P F C$ and ACC}

The general analysis reveals significant activity in the MPFC (BA 9/10) and anterior cingulate cortex (ACC; BA 32) during the mindreading task in the empathizing group, which is not seen in the systemizing group. Although the MPFC, ACC, TPJ and TPs are all deemed important for mindreading, activity in the MPFC and ACC is exemplary of simulation-type processing in the brain $[19,18,27,57,61]$.

Singer et al. [61] and Morrison et al. [51] found shared activity in the ACC for the observation and experience of painful stimuli. Similarly, several fMRI studies indicate that the MPFC is implicated in both first-person (i.e., introspection and self-reflection) and thirdperson mindreading (i.e., theory of mind) (e.g., [48,65]). A study by Ochsner et al. [54] shows that the attribution of emotions to self and other elicits shared neurological activity in the MPFC. Vogeley et al. [65] similarly found shared prefrontal activation for first-person and third-person mental state attribution (i.e., mindreading). Gusnard et al. [30] and Johnson et al. [38] found MPFC activation for introspective judgments and self-reflection. Self-reflective thought activated the anterior regions of the MPFC. Reflecting upon current emotions also activates this region $[30,45]$. In a study by Kelley et al. [40] participants were asked to make judgments about trait adjectives that were either self-relevant, other-relevant or case judgments. Both the self-condition and the other-condition elicited changes in the MPFC. Moreover, Mitchell et al. [50] found activity in a region of the ventral MPFC that correlated with perceived self/other similarity. Last but not least, Frith and Frith [21] mention that out of 12 mindreading studies that they reviewed, all of them include MPFC activation.

\subsubsection{IFG and IPL}

Our ROI analysis reveals stronger activation in the IFG and IPL in the empathizing group that overlaps with areas of activation in human MNS studies, both related to studies comparing individuals with ASCs to normal controls during observation versus imitation of faces [13,53], as well as studies using normal individuals during observation versus imitation of faces $[8,9,24,47,56]$ and gestures $[25,29,31,34]$. It is important to mention that Carr et al. [9] did not find significant differences in activation, both for the observation and imitation task, between whole face stimuli, mouth only stimuli and eyes-only stimuli. Possibly, mouth only stimuli and eyes-only stimuli elicit similar MN activity as whole face stimuli by means of a triggering process (e.g., observation and imitation of eyes-only stimuli likely trigger a whole face mimicry pattern; see [15]).

Overall, our empathizing group activates so-called MN-areas significantly more than our systemizing group, which could imply that the empathizing group relies more on mirroring simulation. Recently, Tamietto et al. [64] found evidence in favor of nonlateralized perceptual processing of social emotions. Hence, the left-lateralized IFG and IPL differences in the empathizing group, likely reflect differences in emotion experience, rather than emotion perception [52]. However, we must mention that our left IPL coordinates also correspond to studies that do not involve typical MNS tasks, such as reflecting upon one's own personality traits [41], first-person perspective taking/simulation [58], and autobiographical memory [46]. Hence, instead of possibly being exemplary of mirroring simulation, the left IPL activation could be exemplary of non-mirroring simulation.

\subsubsection{Temporal lobes}

Our ROI analysis reveals stronger activity in the temporal lobes in the empathizing group that overlaps with areas that are 
implicated in experiential (i.e., 'reliving') autobiographical memory $[12,26]$, autobiographical memory $[17,46]$, self-knowledge retrieval [55], perspective taking/simulation [58], emotional face mirroring [60] and imagined emotion attribution [33]. Possibly, such autobiographical, perspective taking and experiential activation patterns in the empathizing group reflect simulation-related processing in the brain, in which one's own (past) experiential states contribute to mindreading.

\subsection{The systemizing group}

\subsubsection{Temporal lobes}

Goldman [27] hypothesized that individuals that do not rely on simulation-type processing during face-based emotion recognition (e.g., individuals with brain damage) might rely on an alternative 'theorizing' method to solve face-based mindreading tasks. Interestingly, our differential contrast analysis reveals stronger activation in the systemizing group in the left parahippocampal gyrus which might reflect a theorizing approach to face-based mindreading. The parahippocampal gyrus is involved in recollective memory and the encoding/retrieval of associations (for a review, see [63]), and spatial layout encoding involved in associative processing particularly [14].

Our ROI analysis further reveals stronger activation in the temporal lobes that is implicated in associative memory for faces [32], and associative memory versus autobiographical memory tasks [11]. Purely hypothetically, the activation in these temporal areas in the systemizing group might account for a more rule-based or 'theorizing' approach to face-based mindreading in which particular geometric and configural properties of emotional faces are related to particular emotions. A more theorizing-driven approach might compensate for reduced simulation-type processing in these individuals. Similar to our hypothesis in the empathizing group, Schulte-Rüther et al. [60] hypothesize that the subjects in their fMRI study "used their own personal past experiences to gain access to other persons' thoughts and emotional states" (p. 1368). It is not unlikely that the systemizing individuals in our study use their own (past) experiential states less, and focus more on specific facial configurations (i.e., a rule-based approach) to solve the face-based mindreading task.

Our results suggest that simulation-based processes are used in both our groups, which is in line with Goldman's [27] view. In line with recent fMRI studies [44,61], simulation-based processing does appear to be reduced in individuals with low self-reported empathy. Moreover, individuals that are high on systemizing appear to use non-simulation-based processes more strongly compared to individuals that score low on self-reported systemizing. Hence, partially distinct mechanisms for face-based mindreading in normal individuals might exist depending on one's cognitive style. We therefore urge future studies to investigate this possibility.

Although both groups perform very well on the face-based mindreading task, the empathizing individuals score significantly better than the systemizing individuals. This raises the following question: does stronger simulation lead to better mindreading? One potential limitation of this study is the fact that our face-based mindreading task involves eyes-only stimuli. Eyes-only stimuli might favor the empathizing group over the systemizing group regardless of any existing differences in simulation-based processing. In principle, eyes-only stimuli do not obstruct simulation-type processing, since whole face mimicry patterns are triggered by emotional stimuli in general and not just by emotional faces [15]. They might however influence 'rule-based' processing. Eyesonly stimuli provide less overt configural information compared to whole face stimuli, and thus make the task more difficult for the systemizing group if they indeed use more 'rule-based' mechanisms during mindreading. On a separate note, a study by Lahaie et al. [43] found evidence for intact configural processing in individuals with Asperger syndrome - which typically have very high systemizing and very low empathizing skills [1] - along with enhanced processing for individual face parts. Such enhanced processing might compensate for reduced configural information in normal individuals with high systemizing skills.

It has also been argued that individuals with Asperger syndrome process eye/face stimuli differently and that this might, in part, be due to reduced or absent fixation on the eye region [59]. Hence, reduced fixation on the eye stimuli could potentially explain the behavioral difference in our groups. Because our subjects were normal individuals, reduced fixation on the eye region in the systemizing individuals appears, at least at first, to be a less plausible hypothesis. Moreover, the high scores on the mindreading task in both groups in our study show that both groups did attend to the eye stimuli. Nevertheless, we cannot rule out that the systemizing groups fixated less on the stimuli compared to the empathizing group. Indeed, stronger activation in the empathizing group in the FG potentially argues in favor of the latter hypothesis [39]. In sum, several hypotheses are possible: either the empathizing group are better mindreaders due to stronger simulation-based processing in these individuals compared to the systemizing group, or the stimulus material slightly disfavored the systemizing group due to reduced configural information, or the systemizing group fixated less on the stimuli compared to the empathizing group resulting in more false answers. To explore these possibilities, future studies should compare eyes-only and whole face stimuli, and, if possible, incorporate eye-tracking data.

\section{Conclusion}

Face-based mindreading involves simulation-type and simulation-related neural activity in both empathizing and systemizing individuals. Importantly however, the empathizing individuals show more neural activity indicative of simulationbased processes, while the systemizing individuals show more neural activity indicative of non-simulation-based processes, possibly involving stronger rule-based or 'theorizing' mechanisms. Normal individuals might have partially distinct neural mindreading mechanisms depending on their specific cognitive style. Moreover, future research should investigate whether or not stronger simulation leads to better mindreading.

\section{Conflict of interest}

No conflict of interest.

\section{Acknowledgements}

Funding was provided by the National Institutes of Health, NIH R01 NS031443-11. The first author is a Research Fellow of the Research Foundation Flanders.

\section{References}

[1] S. Baron-Cohen, The Essential Difference. The Truth about the Male and Female Brain, Perseus, New York, 2003.

[2] S. Baron-Cohen, T. Jolliffe, C. Mortimore, M. Robertson, Another advanced test of theory of mind: evidence from very high functioning adults with autism or Asperger Syndrome, J. Child Psychol. Psychiatry 38 (1997) 813-822.

[3] S. Baron-Cohen, R.C. Knickmeyer, M.K. Belmonte, Sex differences in the brain: Implications for explaining autism, Science 310 (2005) 819-823.

[4] S. Baron-Cohen, J. Richler, D. Bisarya, N. Gurunathan, S. Wheelwright, The systemizing quotient: an investigation of adults with Asperger syndrome or 
high-functioning autism, and normal sex differences, Philos. Trans. R. Soc. B 358 (2003) 361-374.

[5] S. Baron-Cohen, H.A. Ring, S. Wheelwright, E.T. Bullmore, M.J. Brammer, A. Simmons, S.C.R. Williams, Social intelligence in the normal and autistic brain: an fMRI study, Eur. J. Neurosci. 11 (1999) 1891-1898.

[6] S. Baron-Cohen, S. Wheelwright, The empathy quotient: an investigation of adults with Asperger syndrome or high functioning autism, and normal sex differences, J. Autism Dev. Disord. 34 (2004) 164-175.

[7] S. Baron-Cohen, S. Wheelwright, J. Hill, Y. Raste, I. Plumb, The 'reading the mind in the eyes' test revised version: a study with normal adults and adults with Asperger syndrome or high-functioning autism, J. Child Psychol. Psychiatry 42 (2001) 241-252.

[8] G. Buccino, F. Binkofski, G.R. Fink, L. Fadiga, L. Fogassi, V. Gallese, R.J. Seitz, K. Zilles, G. Rizzolatti, H.-J. Freund, Action observation activates premotor and parietal areas in a somatotopic manner: an fMRI study, Eur. J. Neurosci. 13 (2001) 400-404

[9] L. Carr, M. Iacoboni, M.C. Dubeau, J.C. Mazziotta, G.L. Lenzi, Neural mechanisms of empathy in humans: a relay from neural systems for imitation to limbic areas, Proc. Natl. Acad. Sci. U.S.A. 100 (2003) 5497-5502.

[10] Y. Cheng, L. Po-Lei, C.-Y. Yang, C.P. Lin, D. Hung, J. Decety, Gender differences in the Mu rhythm of the human mirror-neuron system, PLoS One 5 (2008) 1-7.

[11] M.A. Conway, D.J. Turk, S.L. Miller, J. Logan, R.D. Nebes, C.C. Meltzer, J.T. Becker, A positron emission tomography (PET) study of autobiographical memory retrieval, Memory 7 (1999) 679-702.

[12] S.M. Daselaar, H.J. Rice, D.L. Greenberg, R. Cabeza, K.S. LaBar, D.C. Rubin, The spatiotemporal dynamics of autobiographical memory: Neural correlates of recall, emotional intensity, and reliving, Cereb. Cortex 18 (2008) 217-229.

[13] M. Dapretto, M.S. Davies, J.H. Pfeifer, A.A. Scott, M. Sigman, S.Y. Bookheimer M. Iacoboni, Understanding emotions in others: mirror neuron dysfunction in children with autism spectrum disorders, Nat. Neurosci. 9 (2006 28-30.

[14] L. Davachi, Item, context and relational episodic encoding in humans, Curr. Opin. Neurobiol. 16 (2006) 693-700.

[15] U. Dimberg, M. Thunberg, S. Grunedal, Facial reactions to emotional stimuli: automatically controlled emotional responses, Cogn. Emotion 16 (2002) 449-471.

[16] I. Dinstein, J.L. Gardner, M. Jazayeri, D.J. Heeger, Executed and observed movements have different distributed representations in human aIPS, J. Neurosci. 28 (2008) 11231-11239.

[17] G.R. Fink, H.J. Markowitsch, M. Reinkemeier, T. Bruckbauer, J. Kessler, W.D. Heiss, Cerebral representation of one's own past: neural networks in autobiographical memory, J. Neurosci. 16 (1996) 4275-4282.

[18] F. Focquaert, J. Braeckman, S.M. Platek, An evolutionary cognitive neuroscience perspective on human self-awareness and theory of mind, Philos. Psychol. 21 (2008) 47-68.

[19] F. Focquaert, S.M. Platek, Social cognition and the evolution of self-awareness, in: S.M. Platek, J.P. Keenan, T.K. Shackelford (Eds.), Evolutionary Cognitive Neuroscience, MIT Press, Cambridge, MA, 2007, pp. 457-497.

[20] F. Focquaert, M.S. Steven, G.W. Wolford, A. Colden, M.S. Gazzaniga, Empathizing and systemizing cognitive traits in the sciences and humanities, Pers. Indiv. Differ. 43 (2007) 619-625.

[21] U. Frith, C.D. Frith, Development and neurophysiology of mentalizing, Philos. Trans. R. Soc. B 358 (2003) 459-473.

[22] V. Gallese, A. Goldman, Mirror neurons and the simulation theory of mindreading, Trends Cogn. Sci. 2 (1998) 493-501.

[23] M.S. Gazzaniga, The Ethical Brain, Dana Press, New York, 2005.

[24] V. Gazzola, L. Aziz-Zadeh, C. Keysers, Empathy and the somatotopic auditory mirror system in humans, Curr. Biol. 16 (2006) 1824-1829.

[25] E. Gerardin, A. Sirigu, S. Lehéricy, J. Poline, B. Gaymard, C. Marsault, Y. Agid, D. Le Bihan, Partially overlapping neural networks for real and imagined hand movements, Cereb. Cortex 10 (2000) 1093-1104.

[26] A. Gilboa, G. Winocur, C.L. Grady, S.J. Hevenor, M. Moscovitch, Remembering our past: functional neuroanatomy of recollection of recent and very remote personal events, Cereb. Cortex 14 (2004) 1214-1225.

[27] A.I. Goldman, Simulating Minds. The Philosophy, Psychology, and Neuroscience of Mindreading, Oxford University Press, New York, 2006.

[28] A.I. Goldman, N. Sebanz, Simulation, mirroring, and a different argument from error, Trends Cogn. Sci. 9 (2005) 320.

[29] J. Grèzes, J.L. Armony, J. Rowe, R.E. Passingham, Activation related to "mirror" and "canonical" neurons in the human brain: an fMRI study, Neuroimage 18 (2003) 928-937.

[30] D.A. Gusnard, E. Akbudak, G.L. Shulman, M.E. Raichle, Medial prefrontal cortex and self-referential mental activity: relation to a default mode of brain function, Proc. Natl. Acad. Sci. U.S.A. 98 (2001) 4259-4264.

[31] F. Hamzei, M. Rijntjes, C. Dettmers, V. Glauche, C. Weiller, C. Büchel, The human action recognition system and its relationship to Broca's area: an fMRI study, Neuroimage 19 (2003) 637-644.

[32] K. Henke, C.R.A. Mondadori, V. Treyer, R.M. Nitsch, A. Buck, C. Hock, Nonconscious formation and reactivation of semantic associations by way of medical temporal lobes, Neuropsychologia 41 (2003) 863-876.

[33] C.I. Hooker, S.C. Verosky, L.T. Germine, R.T. Knight, M. D’Esposito, Mentalizing about emotion and its relationship, Soc. Cogn. Affect. Neurosci. 3 (2008) 204-217.

[34] M. Iacoboni, R.P. Woods, M. Brass, H. Bekkering, J.C. Mazziotta, G. Rizzolatti, Cortical mechanisms of human imitation, Science 286 (1999) 2526-2528.
[35] M. Jabbi, M. Swart, C. Keysers, Empathy for positive and negative emotions in the gustatory cortex, Neuroimage 34 (2007) 1744-1753.

[36] M. Jenkinson, P.R. Bannister, J.M. Brady, S.M. Smith, Improved optimisation for the robust and accurate linear registration and motion correction of brain images, Neuroimage 17 (2002) 825-841.

[37] M. Jenkinson, S.M. Smith, A global optimisation method for robust affine registration of brain images, Med. Image Anal. 5 (2001) 143-156.

[38] S.C. Johnson, L.C. Baxter, L.S. Wilder, J.G. Pipe, J.E. Heiserman, G.P. Prigatano, Neural correlates of self-reflection, Brain 125 (2002) 1808-1814.

[39] N. Kanwisher, J. McDermott, M.M. Chun, The fusiform face area: a module in human extrastriate cortex specialized for face perception, J. Neurosci. 17 (1997) 4302-4311.

[40] W.M. Kelley, C.N. Macrae, C.L. Wyland, S. Caglar, S. Inati, T.F. Heatherton, Finding the self: an event-related fMRI study, J. Cogn. Neurosci. 14 (2002) 785-794.

[41] T.W. Kjaer, M. Nowak, H.C. Lou, Reflective self-awareness and conscious states: PET evidence for a common midline parietofrontal core, Neuroimage 17 (2002) 1080-1086.

[42] K. Koch, K. Pauly, T. Kellermann, N.Y. Seiferth, M. Reske, V. Backes, T. Stöcker, N.J. Shah, K. Amunts, T. Kircher, F. Schneider, U. Habel, Gender differences in the cognitive control of emotion: an fMRI study, Neuropsychologia 47 (2007) 2744-2754.

[43] A. Lahaie, L. Mottron, A. Arguin, C. Berthiaume, B. Jemel, D. Saumier, Face perception in high-functioning autistic adults: Evidence for superior processing of face parts, not for a configural face-processing deficit, Neuropsychologia 20 (2006) 30-41.

[44] C. Lamm, C.D. Batson, J. Decety, The neural substrate of human empathy: effects of perspective-taking and cognitive appraisal, J. Cogn. Neurosci. 19 (2007) 42-58.

[45] R.D. Lane, E.M. Reiman, M.M. Bradley, P.J. Lang, G.L. Ahern, R.J. Davidson, G.E. Schwartz, Neuroanatomical correlates of pleasant and unpleasant emotion, Neuropsychologia 35 (1997) 1437-1444.

[46] T.M.C. Lee, H. Liu, C.C.H. Chan, S. Mahankali, C. Feng, J. Hou, P.T. Fox, J. Gao, Lie detection by functional magnetic resonance imaging, Hum. Brain Mapp. 15 (2002) 157-164.

[47] K.R. Leslie, S.H. Johnson-Frey, S.T. Grafton, Functional imaging of face and hand imitation: towards a motor theory of empathy, Neuroimage 21 (2004)601-607.

[48] C.N. Macrae, T.F. Heatherton, W.M. Kelley, A self less ordinary: the medial prefrontal cortex and you, in: M.S. Gazzaniga (Ed-in-chief), The Cognitive Neurosciences III, MIT Press, Boston, MA, 2004, pp. 1067-1075.

[49] J.A. Maldjian, P.J. Laurienti, R.A. Kraft, J.H. Burdette, An automated method for neuroanatomic and cytoarchitectonic atlas-based interrogation of fmri data sets, Neuroimage 19 (2003) 1233-1239.

[50] J.P. Mitchell, M.R. Banaji, C.N. Macrae, The link between social cognition and self-referential thought in the medial prefrontal cortex, J. Cogn. Neurosci. 17 (2005) 1306-1315.

[51] I. Morrison, D. Lloyd, G. di Pellegrino, N. Roberts, Vicarious responses to pain in anterior cingulate cortex: is empathy a multisensory issue? Cogn. Affect. Behav. Neurosci. 4 (2004) 270-278.

[52] F.C. Murphy, I. Nimmo-Smith, A.D. Lawrence, Functional neuroanatomy of emotions: a meta-analysis, Cogn. Affect. Behav. Neurosci. 3 (2003) $207-233$.

[53] N. Nishitani, S. Avikainen, R. Hari, Abnormal imitation-related cortical activation sequences in Asperger's syndrome, Ann. Neurol. 55 (2004) 558-562.

[54] K.N. Ochsner, K. Knierim, D.H. Ludlow, J. Hanelin, T. Ramachandran, G. Glover, S.C. Mackey, Reflecting upon feelings: an fMRI study of neural systems supporting the attribution of emotion to self and other, J. Cogn. Neurosci. 16 (2004) 1746-1772.

[55] J.H. Pfeifer, M.D. Lieberman, M. Dapretto, I know you are but what am I? Neural bases of self- and social knowledge retrieval in children and adults, J. Cogn. Neurosci. 19 (2007) 1323-1337.

[56] J.H. Pfeifer, M. Iacoboni, J.C. Mazziotta, M. Dapretto, Mirroring others' emotions relates to empathy and interpersonal competence in children, Neuroimage 39 (2008) 2076-2085.

[57] S.M. Platek, J.P. Keenan, G.G. Gallup Jr., F.B. Mohamed, Where am I? The neurological correlates of self and other, Cogn. Brain Res. 19 (2004) 114-122.

[58] P. Ruby, J. Decety, Effect of subjective perspective taking during simulation of action: a PET investigation of agency, Nat. Neurosci. 4 (2001) 546-550.

[59] M.D. Rutherford, K.A. Clements, A.B. Sekuler, Differences in discrimination of eye and mouth displacement in autism spectrum disorders, Vision Res. 47 (2007) 2099-2110.

[60] M. Schulte-Rüther, H.J. Markowitsch, G.R. Fink, M. Piefke, Mirror neuron and theory of mind mechanisms involved in face-to-face interactions: a functional magnetic resonance imaging approach to empathy, J. Cogn. Neurosci. 19 (2007) 1354-1372.

[61] T. Singer, B. Seymour, J. O’Doherty, H. Kaube, R.J. Dolan, C.D. Frith, Empathy for pain involves the affective but not sensory components of pain, Science 303 (2004) 1157-1162.

[62] S.M. Smith, M. Jenkinson, M.W. Woolrich, C.F. Beckmann, T.E.J. Behrens, H. Johansen-Berg, P.R. Bannister, M. De Luca, I. Drobnjak, D.E. Flitney, R. Niazy, J. Saunders, J. Vickers, Y. Zhang, N. De Stefano, J.M. Brady, P.M. Matthews, Advances in functional and structural MR image analysis and implementation as FSL, Neuroimage 23/S1 (2004) 208-219.

[63] L.R. Squire, C.E.L. Starck, R.E. Clarck, The medial temporal lobe, Annu. Rev. Neurosci. 27 (2004) 279-306. 
[64] M. Tamietto, M. Adenzato, G. Geminiani, B. de Gelder, Fast recognition of social emotions takes the whole brain: interhemispheric cooperation in the absence of cerebral asymmetry, Neuropsychologia 45 (2007) 836-843.

[65] K. Vogeley, P. Bussfeld, A. Newen, S. Herrmann, F. Happé, P. Falkai, W. Maier N.J. Shah, G.R. Fink, K. Zilles, Mind reading: neural mechanisms of theory of mind and self-perspective, Neuroimage 14 (2001) 170-181.
[66] M.W. Woolrich, B.D. Ripley, J.M. Brady, S.M. Smith, Temporal autocorrelation in univariate linear modelling of fMRI data, Neuroimage 14 (2001) 1370-1386.

[67] K.J. Worsley, A.C. Evans, S. Marrett, P. Neelin, A three-dimensional statistical analysis for CBF activation studies in human brain, J. Cerebr. Blood Flow Metab. 12 (1992) 900-918. 